\title{
Holographic heat current as Noether current
}

\author{
Hai-Shan Liu, ${ }^{a}$ H. Lï̈ ${ }^{b}$ and C.N. Pope ${ }^{c, d}$ \\ ${ }^{a}$ Institute for Advanced Physics 8 Mathematics, Zhejiang University of Technology, \\ Hangzhou 310023, China \\ ${ }^{b}$ Center for Advanced Quantum Studies, Department of Physics, Beijing Normal University, \\ Beijing 100875, China \\ ${ }^{c}$ George P. $\&$ Cynthia Woods Mitchell Institute for Fundamental Physics and Astronomy, \\ Texas A\&M University, \\ College Station, TX 77843, U.S.A. \\ ${ }^{d}$ DAMTP, Centre for Mathematical Sciences, Cambridge University, \\ Wilberforce Road, Cambridge CB3 OWA, U.K. \\ E-mail: hsliu.zju@gmail.com, mrhonglu@gmail.com, pope@physics.tamu.edu
}

ABSTRACT: We employ the Noether procedure to derive a general formula for the radially conserved heat current in AdS planar black holes with certain transverse and traceless perturbations, for a general class of gravity theories. For Einstein gravity, the general higherorder Lovelock gravities and also a class of Horndeski gravities, we derive the boundary stress tensor and show that the resulting boundary heat current matches precisely the bulk Noether current.

KEYwords: AdS-CFT Correspondence, Holography and condensed matter physics $(\mathrm{AdS} / \mathrm{CMT})$

ARXIV EPRINT: 1708.02329 


\section{Contents}

1 Introduction 1

2 Holographic heat current from Noether procedure 2

2.1 AdS planar black holes and a linear perturbation 2

2.2 Radially conserved current 3

2.3 Holographic heat current 5

3 Holographic heat current in EMA theory 5

4 Holographic heat current in general Lovelock gravities $\quad 7$

$\begin{array}{ll}4.1 \text { Bulk theory and conserved current } & 7\end{array}$

$\begin{array}{llr}4.2 & \text { Surface terms for the Lovelock actions } & 9\end{array}$

$\begin{array}{lll}4.3 & \text { Flat-boundary counterterms for Lovelock actions } & 11\end{array}$

$\begin{array}{lll}4.4 & \text { Boundary energy-momentum tensor } & 12\end{array}$

5 Holographic heat current in Horndeski gravities $\quad 14$

$\begin{array}{llr}6 & \text { Conclusions } & 17\end{array}$

\section{Introduction}

Recently, gauge/gravity duality has been used to understand various phenomena for strongly coupled systems in condensed matter physics [1-4]. In particular, holographic gravity models with momentum relaxation have attracted much attention. These models can serve as a realistic description of materials with impurities [5-28].

With the help of gauge/gravity duality, one can calculate the transport coefficients in these strongly coupled systems by analysing the linear response to a small perturbation around a black hole background which describes an equilibrium state. Among these transport coefficients, much effort has been directed to calculating the AC conductivity, mostly involving the use of numerical methods. However, ways to calculate the DC conductivity analytically have also been developed, based on the "membrane paradigm" [29]. The key step involves constructing a radially conserved current [10], which provides an analytical relation between the holographic boundary information and the black hole horizon data.

With the same philosophy, one can obtain the holographic thermal and thermoelectric conductivities. This was developed in [15] for the case of Einstein-Maxwell-Dilaton (EMD) gravity, by manipulating the equations of motion to construct a radially conserved bulk heat current. However, unlike the electric current which arises naturally from the Maxwell equation, the bulk heat current is considerably more subtle to calculate, since it makes use of both the Einstein and Maxwell equations. The problem can be further exacerbated in 
higher-order gravity theories. Matching the bulk current to the boundary heat current, derived from the boundary stress tensor, can also be quite involved. A general procedure for constructing the holographic electric and heat currents for higher derivative gravity by using dimension reduction was proposed in [26]. However, a general and simple formula for this bulk current is lacking in literature; the known examples were obtained for certain specific theories. The first example was given by Donos and Gauntlett [15] for the EMD theory. Another notable example is the holographic heat current in Einstein-Gauss-Bonnet gravity $[19,26]$.

The purpose of this paper is to present a general formula for such radially conserved currents associated with certain transverse and traceless (TT) perturbation of the AdS planar black hole. We follow closely the procedure described by Wald [31, 32], and show that the radially conserved current is simply the Noether current associated with the timelike Killing vector. We then show that the Noether current indeed matches the boundary heat current for AdS planar black holes in Einstein gravity, general Lovelock gravities [33] and also a class of Horndeski gravities [34].

The paper is organized as follows. In section 2, we consider a general class of gravity theories and use the Noether procedure to derive a general formula for the radially conserved current. In section 3, we consider the simple example of Einstein-Maxwell-Axion (EMA) theory, for which we derive the boundary stress tensor and show that the bulk current and boundary heat current match precisely. In sections 4 and 5, we consider general Lovelock gravities and a class of Horndeski gravities respectively. We derive the boundary heat currents and show that they again match precisely with the corresponding radially conserved current. We conclude the paper in section 6 .

\section{Holographic heat current from Noether procedure}

\subsection{AdS planar black holes and a linear perturbation}

In this paper, we shall consider a general class of gravity theories in $n$ dimensions, coupled to a set of matter fields including a Maxwell field. We shall assume that the theory admits an AdS spacetime vacuum and that the full action takes the form

$$
S=\int_{\mathcal{M}} d^{n} x \sqrt{-g} L\left(g_{\mu \nu}, A_{\mu}, \phi\right)+\int_{\partial \mathcal{M}} d^{n-1} x \sqrt{-h}\left(L_{\mathrm{surf}}+L_{\mathrm{ct}}\right),
$$

where $A_{\mu}$ is the Maxwell field and $\phi$ denotes any additional matter fields in the theory. $L_{\text {surf }}$ is the Gibbons-Hawking term or its generalization, and $L_{\text {ct }}$ denotes the holographic counterterms. For simplicity, we shall assume that the Maxwell field is minimally coupled to gravity, as in the case of the EMD theory.

We shall be considering static background metrics of the form

$$
d s^{2}=-\tilde{f} d t^{2}+\frac{d r^{2}}{f}+g^{2} r^{2} d x^{i} d x^{i},
$$

where $\tilde{f}$ and $f$ are functions of $r$, which at large $r$ approach the forms

$$
\tilde{f}(r)=g^{2} r^{2}+\cdots, \quad f(r)=g^{2} r^{2}+\cdots,
$$


where the ellipses denote terms of lower order in $r$. Thus the metrics are asymptotic to $\mathrm{AdS}_{n}$ spacetime with $R_{\mu \nu}=-(n-1) g^{2} g_{\mu \nu}$. (Note that in this paper $g=1 / \ell$ is the inverse of the radius of the AdS, and it should not be confused with the determinant of the metric.) Also the coordinates $x_{i}$ have the same (engineering) dimension as the time coordinate $t$, namely (Length) ${ }^{1}$. For the purpose of calculating the heat current, we shall consider transverse traceless (TT) metric perturbations for which the metric (2.2) takes the form

$$
d s^{2}=-\tilde{f} d t^{2}+\frac{d r^{2}}{f}+g^{2} r^{2} d x^{i} d x^{i}+2 \delta g_{t x_{1}} d t d x_{1},
$$

with $\delta g_{t x_{1}}$ being infinitesimal, and (in principle) depending on all the coordinates. However, for our purpose, we follow [15] that the perturbation depends on $r$ only, except for a linear time dependence that is necessary for satisfying the ingoing boundary condition on the black hole horizon. The analogous strategy was developed for holographic DC currents [14]. Note that we did not include a perturbation $\delta g_{r x_{1}}(r)$, since it can be can be removed by means of a coordinate transformation.

\subsection{Radially conserved current}

Our derivation of a radially conserved current will closely follow the procedure described by Wald [31, 32]. Our starting point is a bulk Lagrangian density $\mathcal{L}$, written in terms of a scale quantity $L_{0}$ as

$$
\mathcal{L}=\sqrt{-g} L_{0} .
$$

Under variations of the fields $\delta \mathcal{L}$ takes the form

$$
\delta \mathcal{L}=E . O . M .+\sqrt{-g} \nabla_{\mu} J^{\mu}\left(\delta g_{\mu \nu}, \delta \phi, \delta A_{\mu}\right) .
$$

where E.O.M. denotes the equations of motion, and the additional term collects together the various total derivatives that do not contribute to the equations of motion.

In the Wald procedure, the two $(n-1)$-forms $\Theta_{(n-1)}$ and $J_{(n-1)}$ are defined, by

$$
\Theta_{(n-1)}=* J_{(1)}, \quad J_{(n-1)}=\Theta_{(n-1)}-\xi_{(1)} \cdot * L_{0},
$$

where $J_{(1)}=J^{\mu} g_{\mu \nu} d x^{\nu}$, and $\xi_{(1)}=\xi^{\mu} g_{\mu \nu} d x^{\nu}$ denotes the 1-form associated with an infinitesimal diffeormorphism $\delta x^{\mu}=\xi^{\mu}$. The term $\xi_{(1)} \cdot * L_{0}$ is the $(n-1)$ form obtained by contracting $\xi$ onto the $n$-form $* L_{0}$. The $(n-1)$ form $J_{(n-1)}$ can be written as

$$
J_{(n-1)}=\Theta_{(n-1)}-\xi_{(1)} \cdot * L_{0}=d * J_{(2)}+\text { E.O.M }
$$

where the gravity contribution to $J_{(2)}$ is

$$
J_{(2) \mathrm{gr}}^{\mu \nu}=2 \frac{\partial L}{\partial R_{\mu \nu \rho \sigma}} \nabla_{\rho} \xi_{\sigma}+4 \xi_{\rho} \nabla_{\sigma} \frac{\partial L}{\partial R_{\mu \nu \rho \sigma}} .
$$

It is worth noting that for the Einstein-Hilbert term, we have simply $J_{(2) \text { gr }}^{\mu \nu}=2 \nabla^{\mu} \xi^{\nu}$. Note also that the contribution to $J_{(2)}$ from the minimally-coupled Maxwell field is

$$
J_{(2) A}^{\mu \nu}=\xi^{\rho} A_{\rho} F^{\mu \nu} .
$$


On shell, the formula (2.8) turns out to be

$$
d * J_{(2)}=J_{(n-1)}=\Theta_{(n-1)}-\xi_{(1)} \cdot * L_{0} .
$$

The Hodge dual of this equation is

$$
* d * J_{(2)}=* J_{(n-1)}=* \Theta_{(n-1)}-*\left(\xi_{(1)} \cdot * L_{0}\right),
$$

which gives

$$
\frac{1}{\sqrt{-g}} \partial_{\nu}\left(\sqrt{-g} J_{(2)}^{\mu \nu}\right)=J_{(1)}^{\mu}\left(\delta g_{\mu \nu}, \delta \phi, \delta A_{\mu}\right)+L_{0} \xi^{\mu} .
$$

If we consider a static metric, with $\xi$ taken to be the timelike Killing vector $\xi^{\mu} \partial_{\mu}=$ $\partial / \partial t$, we have

$$
\delta g_{\mu \nu}=\nabla_{\mu} \xi_{\nu}+\nabla_{\nu} \xi_{\mu}=0, \quad \delta A_{\mu}=\xi^{\nu} \partial_{\nu} A_{\mu}+\left(\partial_{\mu} \xi^{\nu}\right) A_{\nu}=0, \quad \delta \phi=\xi^{\mu} \nabla_{\mu} \phi=0
$$

which implies $J_{(1)}^{\mu}=0$. Thus we have

$$
\frac{1}{\sqrt{-g}} \partial_{\nu}\left(\sqrt{-g} J_{(2)}^{\mu \nu}\right)=L_{0} \xi^{\mu}
$$

The components of the right-hand side are all zero except in the $t$ direction, and so in particular we have the radial conservation for the components $J_{(2)}^{\mu \nu}$,

$$
\frac{1}{\sqrt{-g}} \partial_{r}\left(\sqrt{-g} J_{(2)}^{x_{i} r}\right)=0
$$

where $\mu=x_{i}$ represents coordinate index values in the directions of the spatial boundary metric, and $\nu=r$ denotes the radial index direction. Thus we obtain a radially conserved current

$$
\mathcal{J}^{x_{i}}=\sqrt{-g} J_{(2)}^{r x_{i}}
$$

In particular, the gravity and Maxwell field contributions to the 2-form are given by (2.9) and (2.10) respectively.

It is worth remarking that the bulk Noether current (2.17) is radially conserved for all static or stationary solutions, where $\partial / \partial t$ is a Killing vector. For the static backgrounds we are focusing on in this paper, this Noether current vanishes identically for the static background configuration itself, and it gives rise to a non-trivial result for the metric (2.4), where there is a TT perturbation. The linear time dependence in $\delta g_{t x_{1}}$ discussed under (2.4) will not affect that $\mathcal{J}^{x_{1}}$ is radially conserved at the linear level. In the next subsection, we argue that the current (2.17) associated with the perturbation in (2.4) is precisely the bulk dual to the holographic heat current of the boundary field theory of the AdS spacetime. It is worth emphasising that this method can be generalised to include other Killing vectors, such as $\partial_{x_{i}}$, in which case the radially conserved current is related to the $x_{i} x_{j}$ component of the boundary stress tensor, and can be used to calculate the holographic shear viscosity [30]. 


\subsection{Holographic heat current}

Turning on a small electric field $E_{i}$ and thermal gradient $\nabla_{i} T$ will generate an electric current $J^{i}$ and thermal current $Q^{i}=T^{t i}-\mu J^{i}$, where $T^{a b}$ is the boundary stress tensor, $i$ denotes the spatial boundary directions and $\mu$ is the chemical potential. To first order, the generalised Ohm law is given by

$$
\left(\begin{array}{c}
J \\
Q
\end{array}\right)=\left(\begin{array}{cc}
\sigma & \alpha T \\
\bar{\alpha} T & \kappa T
\end{array}\right)\left(\begin{array}{c}
E \\
-(\nabla T) / T
\end{array}\right),
$$

where $\sigma$ is the electric conductivity, $\bar{\kappa}$ is the thermal conductivity and $\alpha, \bar{\alpha}$ are thermoelectric conductivities.

The goal of this paper is to examine a variety of gravity theories, and to establish that the radially-conserved bulk current $\mathcal{J}^{x_{i}}$ in (2.17) matches to the thermal current $Q^{i}$ on the boundary, namely

$$
\left.\mathcal{J}^{x_{i}}\right|_{\text {boundary }}=Q^{i}
$$

Since $\mathcal{J}^{x_{i}}$ is radially conserved, one can read off $\mathcal{J}^{x_{i}}$ using the horizon data, which can be obtained even in the case where an exact solution is not known. It is clear that for the static background (2.2) both the left and right-hand sides of the equation (2.19) vanish, and hence in this case the equality holds trivially. Our goal therefore is to show that the equality (2.19) holds nontrivially, at the linearised level, for the TT perturbation $\delta g_{t x_{1}}$ in (2.4).

In the existing literature, the construction of the radially-conserved current $\mathcal{J}$ was carried out on the case by case basis. The first example was given in [15] for the EMD theory, where the equality (2.19) was also established. Analogous results were also obtained for the Einstein-Gauss-Bonnet theory, in [19].

Our construction of the radially-conserved current through the Noether procedure in section 2 makes it straightforward to construct the bulk current for a variety of theories. It is still necessary to obtain the corresponding boundary thermal current and then to establish the equality (2.19). In section 3, we review this analysis for the Einstein-Maxwell-Axion (EMA) theory. In sections 4, 5, we establish (2.19) for general Lovelock gravities and also a class of Horndeski gravities.

\section{Holographic heat current in EMA theory}

In this section, we review the analysis of the holographic currents in the EMA theory. This allows us to establish notation, and to relate the abstract bulk Noether current in the previous section to the boundary quantities. The Lagrangian is given by

$$
\mathcal{L}=\sqrt{-g}\left(R-2 \Lambda-\frac{1}{4} F^{2}-\frac{1}{2} \sum_{i=1}^{n-2}\left(\partial \chi_{i}\right)^{2}\right),
$$

where $F=d A$. The axions $\chi_{i}$ 's are introduced to provide momentum dissipation, so that one can obtain finite results for the DC conductivity and the thermal heat conductivity. We shall consider the static metric ansatz (2.2), together with

$$
A=a(r) d t, \quad \chi_{i}=\lambda x_{i} .
$$


Note that this is a simpler version of the more general EMD theory discussed in [15]. Charged AdS black holes were constructed in [13]. The focus of this section is to establish the equality (2.19) for this theory, rather than to obtain the explicit result for the heat conductivity, which is a special case of [15]. It follows that the axions do not play any significant role in our discussion. They however must be included in this set to obtain a finite conductivity, since they are responsible for the momentum dissipation [13].

We now consider a perturbation along one spatial direction, say $x_{1}$, with $\delta g_{t x_{1}}, \delta A_{x_{1}}$ and $\delta \chi_{1}$ turned on.

For simplicity, here and after, we consider only static backgrounds, even though the construction of bulk currents in the previous section can be applied to all stationary backgrounds with a time-like Killing vector. There are two radially-conserved currents associated with the perturbation. One is the electric current of the Maxwell field,

$$
J=\sqrt{-g} F^{r x_{1}} .
$$

After imposing boundary conditions on the perturbation, one can evaluate the electric current on the horizon and then obtain the corresponding electric conductivity [13].

One can go one step further to study the holographic thermal conductivity, since there exists an additional radially-conserved Noether current, following the discussion of section 2 . It is given by

$$
\mathcal{J}^{x_{1}}=\sqrt{-g}\left(2 \nabla^{r} \xi^{x_{1}}+a F^{r x_{1}}\right) .
$$

Note that the first term in the bracket comes from the contribution of the Einstein-Hilbert term, and the second comes from the contribution of the Maxwell field. For the AdS planar black hole background with the diagonal metric, it is clear that the Noether current (3.4) vanishes identically. However, for the aforementioned perturbation the current (3.4) is non-vanishing, and can be evaluated at the linear level. We are interested in showing that this current matches with the heat current on the boundary at $r \rightarrow \infty$. The large- $r$ leading falloff at the asymptotic boundary for $\mathcal{J}^{x_{1}}$ is given by

$$
\mathcal{J}_{\text {lin }}^{x_{1}}=(g r)^{n-2}\left(\frac{r \delta g_{t x_{1}}^{\prime}-2 \delta g_{t x_{1}}}{r}-\mu F^{r x_{1}}\right) .
$$

Note that we have imposed the gauge condition $a(r)=0$ on the horizon and so the value of $a(r)$ at infinity is the chemical potential, $a(\infty)=\mu$.

We now turn our attention to the boundary conserved currents. Including the constant counterterm contribution, the boundary stress tensor is given by

$$
T^{a b}=2\left(K h^{a b}-K^{a b}\right)-2(n-2) g h^{a b},
$$

where $h^{a b}$ is the induced boundary metric and $K^{a b}$ is the extrinsic curvature of the boundary. (Since the boundary spacetime is flat, there are no counterterms associated with boundary curvature. Note also that $g=1 / \ell$ here is the inverse of the AdS radius, and should not be confused with the determinant of the metric.)

Specific to our perturbations, we have

$$
T_{\text {lin }}^{t x_{1}}=\frac{r \delta g_{t x_{1}}^{\prime}-2 \delta g_{t x_{1}}}{g^{3} r^{4}},
$$


where a prime denotes the derivative respect to $r$. The boundary electric current along the $x_{1}$ direction is given, to linear order, by

$$
J_{\mathrm{BD}}^{e}=-n_{\mu} F^{\mu x_{1}}=-\frac{1}{g r} F^{r x_{1}} .
$$

The boundary heat current in the presence of the electric current is defined as

$$
Q=(g r)^{n+1} T^{t x_{1}}+(g r)^{n-1} \mu J_{\mathrm{BD}}^{e} .
$$

At linear order in the perturbation, it is given by

$$
Q_{\text {lin }}=(g r)^{n-2}\left(\frac{r \delta g_{t x_{1}}^{\prime}-2 \delta g_{t x_{1}}}{r}-\mu F^{r x_{1}}\right)
$$

Thus we see that the radially-conserved Noether current (3.5) matches precisely to the thermal current on the boundary.

\section{Holographic heat current in general Lovelock gravities}

We begin this section by setting up our conventions for the Lovelock Lagrangian [33], and deriving the form of the radially conserved 2-form current. We then go on to calculate the energy-momentum tensor in the boundary theory, and to show that it is indeed related to the radially conserved 2 -form current. As was seen in previous section, holographic heat currents can be discussed in the pure gravity sector without the inclusion of the Maxwell field. However, it is necessary to include also a mechanism for momentum dissipation, in order to obtain a finite conductivity. In this section, we shall employ free axions spanning the AdS planar directions in order to generate the momentum dissipation [13], as discussed in section 3. As we have seen in section 3 , the axions give no contribution to $J_{(2)}^{\mu \nu}$. Furthermore, their overall contribution to the metric background profile functions is to add a constant to $f$ and $\tilde{f}$ in the large $r$ expansion, without altering the asymptotic form, which remains $f \sim \tilde{f} \sim g^{2} r^{2}$. It follows that they play no rôle in the matching of the bulk currents and boundary stress tensor at asymptotic infinity.

\subsection{Bulk theory and conserved current}

The general bulk Lovelock action is given by

$$
S_{\text {bulk }}=\int d^{n} x \sqrt{-g} L, \quad L=\sum_{k \geq 0} a_{(k)} E_{(k)},
$$

where we define

$$
E_{(k)}=\frac{(2 k) !}{2^{k}} \delta_{\nu_{1} \cdots \nu_{2 k}}^{\mu_{1} \cdots \mu_{2 k}} R_{\mu_{1} \mu_{2}}^{\nu_{1} \nu_{2}} R_{\mu_{3} \mu_{4}}^{\nu_{3} \nu_{4}} \cdots R_{\mu_{2 k-1} \mu_{2 k}}^{\nu_{2 k-1} \nu_{2 k}} .
$$

Note that our multi-index Kronecker delta symbol is defined to have unit strength, so

$$
\delta_{\nu_{1} \cdots \nu_{2 k}}^{\mu_{1} \cdots \mu_{2 k}}=\delta_{\nu_{1}}^{\left[\mu_{1}\right.} \delta_{\nu_{2}}^{\mu_{2}} \cdots \delta_{\nu_{2 k}}^{\left.\mu_{2 k}\right]}
$$


where the square brackets denote conventional unit-strength antisymmetrisations (so, for example, $X^{\left[\mu_{1} \cdots \mu_{p}\right]}=X^{\left[\left[\mu_{1} \cdots \mu_{p}\right]\right]}$ ). Note that with our choice of normalisation for the Lovelock terms $E_{(k)}$, we have

$$
E_{(k)}=R^{k}+\cdots,
$$

with unit coefficient for the purely Ricci-scalar term, where the ellipses denote all terms involving one or more uncontracted Ricci tensor or Riemann tensor. (So, in particular, $E_{(0)}=1, E_{(1)}=R$ and $E_{(2)}=R^{2}-4 R^{\mu \nu} R_{\mu \nu}+R^{\mu \nu \rho \sigma} R_{\mu \nu \rho \sigma}$, etc.) The contribution to the Einstein equation from the $k$ 'th Lovelock term is given by

$$
E_{\nu}^{(k) \mu}=-\frac{(2 k+1) !}{2^{k+1}} \delta_{\nu \nu_{1} \cdots \nu_{2 k}}^{\mu \mu_{1} \cdots \mu_{2 k}} R_{\mu_{1} \mu_{2}}^{\nu_{1} \nu_{2}} \cdots R_{\mu_{2 k-1} \mu_{2 k}}^{\nu_{2 k-1} \nu_{2 k}}
$$

Calculating the conserved current for the $k^{\prime}$ th Lovelock term, using (2.9), we find

$$
J^{(k) \mu \nu}=\frac{a_{(k)} k(2 k) !}{2^{k-1}} \delta_{\rho \sigma \beta_{1} \cdots \beta_{2 k-2}}^{\mu \nu \alpha_{1} \cdots \alpha_{2 k-2}} R_{\alpha_{1} \alpha_{2}}^{\beta_{1} \beta_{2}} \cdots R_{\alpha_{2 k-3} \alpha_{2 k-2}}^{\beta_{2 k-3} \beta_{2 k-2}} \nabla^{\rho} \xi^{\sigma} .
$$

(Note that the second term in (2.9) does not contribute, by virtue of the Bianchi identities for the Riemann tensor.)

We are interested in particular in calculating the linearised contribution to $J^{(k) \mu \nu}$ resulting from the metric perturbation given in (2.4). We find

$$
\begin{aligned}
J_{\text {lin }}^{(k) \mu \nu}= & \frac{a_{(k)} k(k-1)(2 k) !}{2^{k-1}} \delta_{\rho \sigma \beta_{1} \cdots \beta_{2 k-2}}^{\mu \nu \alpha_{1} \cdots \alpha_{2 k-2}} \bar{R}_{\alpha_{1} \alpha_{2}}^{\beta_{1} \beta_{2}} \cdots \bar{R}_{\alpha_{2 k-5} \alpha_{2 k-4}}^{\beta_{2 k-5} \beta_{2 k-4}} \delta R_{\alpha_{2 k-3} \alpha_{2 k-2}}^{\beta_{2 k-3} \beta_{2 k-2}} \bar{\nabla}^{\rho} \xi^{\sigma} \\
& +\frac{a_{(k)} k(2 k) !}{2^{k-1}} \delta_{\rho \sigma \beta_{1} \cdots \beta_{2 k-2}}^{\mu \nu \alpha_{1} \cdots \alpha_{2 k-2}} \bar{R}_{\alpha_{1} \alpha_{2}}^{\beta_{1} \beta_{2}} \cdots \bar{R}_{\alpha_{2 k-3} \alpha_{2 k-2}}^{\beta_{2 k-3} \beta_{2 k-2}} \delta \nabla^{\rho} \xi^{\sigma}
\end{aligned}
$$

where the barred quantities are calculated in the background of the unperturbed metric $(2.2)$.

We now wish to evaluate the linearised contribution $\delta J^{(k) r x_{1}}$ in the limit that $r$ goes to infinity, in order to compare it with the heat current calculated in the boundary theory by evaluating the $t x_{1}$ component of the boundary stress tensor. We may evaluate (4.7) in the large- $r$ limit by simply allowing the metric functions $h$ and $f$ to take their asymptotic forms $\tilde{f}=f=g^{2} r^{2}$. In this limit the background metric is exactly $\mathrm{AdS}_{n}$, and so in particular we have

$$
\bar{R}_{\rho \sigma}^{\mu \nu}=-2 g^{2} \delta_{\rho \sigma}^{\mu \nu}
$$

implying that (4.7) becomes

$$
\begin{aligned}
J_{\operatorname{lin}}^{(k) \mu \nu}= & \frac{12(-1)^{k} a_{(k)} k(k-1)(n-4) ! g^{2 k-4}}{(n-2 k) !} \delta_{\rho \sigma \beta_{1} \beta_{2}}^{\mu \nu \alpha_{1} \alpha_{2}} \delta R_{\alpha_{1} \alpha_{2}}^{\beta_{1} \beta_{2}} \bar{\nabla}^{\rho} \xi^{\sigma} \\
& -\frac{2 k(-1)^{k} a_{(k)}(n-2) ! g^{2 k-2}}{(n-2 k) !} \delta_{\rho \sigma}^{\mu \nu} \delta \nabla^{\rho} \xi^{\sigma} .
\end{aligned}
$$

We also have $\bar{\nabla}^{r} \xi^{t}=-\bar{\nabla}^{t} \xi^{r}=g^{2} r$, and after some algebra we find that

$$
J_{\operatorname{lin}}^{(k) r x_{1}}=\frac{(-1)^{k-1} a_{(k)} k(n-3) ! g^{2 k-2}}{(n-2 k-1) !}\left(\delta g_{t x_{1}}^{\prime}-\frac{2}{r} \delta g_{t x_{1}}\right),
$$


where a prime denotes a derivative with respect to $r$. The radially conserved current is given by $\mathcal{J}^{x_{1}}=\sqrt{-g} J^{r x_{1}}$, which vanishes on the background with the diagonal metric. For the TT perturbation (2.4), the radially-conserved Noether current associated with $\delta g_{t x_{1}}$, evaluated at large $r$, is given by

$$
\begin{aligned}
\mathcal{J}_{\text {lin }}^{x_{1}} & =\sqrt{-\bar{g}} \sum_{k \geq 1} J_{\text {lin }}^{(k) r x_{1}} \\
& =(g r)^{n-2} \sum_{k \geq 1} \frac{(-1)^{k-1} a_{(k)} k(n-3) ! g^{2 k-2}}{(n-2 k-1) !}\left(\delta g_{t x_{1}}^{\prime}-\frac{2}{r} \delta g_{t x_{1}}\right) .
\end{aligned}
$$

\subsection{Surface terms for the Lovelock actions}

Just as in ordinary Einstein gravity, in order to write the theory in such a way that it has a well-defined Hamiltonian formulation, it is necessary to add a surface term to the action that removes the $\nabla \delta g_{\mu \nu}$ terms arising in the variational principle. For Einstein gravity, the necessary boundary term, which was derived by York [35] and by Gibbons and Hawking [36], involves the trace of the second fundamental form on the boundary. A general discussion of the analogous surface terms for general gravity theories was given in [37]. Their discussion made use of an auxiliary field formulation, and after restating it in terms of just the original metric formulation, it may be expressed as follows. We begin by defining a surface action $\widetilde{S}_{\text {surf }}$ as

$$
S_{\text {surf }}=4 \int \sqrt{-h} d^{n-1} x \Psi^{\mu \nu} K_{\mu \nu},
$$

where $K_{\mu_{\nu}}=h_{\mu}{ }^{\rho} \nabla_{\rho} n_{\nu}$ is the second fundamental form, $h_{\mu \nu}=g_{\mu \nu}-n_{\mu} n_{\nu}$ and $n^{\mu}$ is the unit normal vector on the boundary (spacelike, with $n=f^{1 / 2} \partial / \partial r$ in our case). Eventually, the auxiliary field $\Psi^{\mu \nu}$ is solved for, and is given by

$$
\Psi^{\mu \nu}=\frac{\partial L}{\partial R_{\mu \rho \nu \sigma}} n_{\rho} n_{\sigma}
$$

Varying $S_{\text {surf }}$ with respect to the boundary metric to obtain the boundary contributions that will subtract those coming from the integrations by parts for the bulk action, one leaves $\Psi^{\mu \nu}$ unvaried and only then makes the substitution (4.13).

One can instead construct $S_{\text {surf }}$ directly as follows. We begin by defining

$$
\widetilde{S}_{\text {surf }}=4 \int \sqrt{-h} d^{n-1} x \frac{\partial L}{\partial R_{\rho \sigma}^{\mu \nu}} K_{\rho}^{\mu} n^{\nu} n_{\sigma}
$$

Using the Gauss-Codacci equation

$$
R_{c d}^{a b}=\mathcal{R}_{c d}^{a b}-2 K_{[c}^{a} K_{d]}^{b}
$$

we substitute into the expression for $\widetilde{S}_{\text {surf }}$. We may then calculate the desired surface term $S_{\text {surf }}$ by means of the equation

$$
K_{a b} \frac{\partial S_{\text {surf }}}{\partial K_{a b}}=\widetilde{S}_{\text {surf }}
$$


By this means, one is compensating for the fact that (4.14) contains higher powers of $K$, which, when varied, would give too large a contribution in the variation. We can express the solution to (4.16) in the integral form

$$
S\left(\mathcal{R}_{c d}^{a b}, K_{b}^{a}\right)_{\text {surf }}=\int_{0}^{1} \widetilde{S}\left(\mathcal{R}_{c d}^{a b}, u K_{b}^{a}\right)_{\text {surf }} \frac{d u}{u} .
$$

The result of solving (4.16) is very easy to state in the case where we consider the $k$ th Lovelock Lagrangian and where we also take the boundary to be flat (as in our discussion in this paper). We then have simply

$$
S_{\mathrm{surf}}^{(k)}=\frac{1}{2 k-1} \widetilde{S}_{\mathrm{surf}}^{(k)}
$$

and so

$$
\begin{aligned}
S_{\mathrm{surf}}^{(k)} & =\int \sqrt{-h} d^{n-1} x L_{\mathrm{surf}}^{(k)}, \\
L_{\mathrm{surf}}^{(k)} & =\frac{(-1)^{k-1}(2 k) ! a_{(k)}}{2 k-1} \delta_{b_{1} \cdots b_{2 k-1}}^{a_{1} \cdots a_{2 k-1}} K_{a_{1}}^{b_{1}} K_{a_{2}}^{b_{2}} \cdots K_{a_{2 k-1}}^{b_{2 k-1}}
\end{aligned}
$$

If we consider the case where the boundary metric is curved, then from (4.17) we have:

$$
\begin{aligned}
L_{\mathrm{surf}}^{(k)}= & \frac{(2 k) ! a_{(k)}}{2^{k-1}} \delta_{b_{1} \cdots b_{2 k-1}}^{a_{1} \cdots a^{2 k-1}} \times \\
& \int_{0}^{1} d u\left(\mathcal{R}_{a_{1} a_{2}}^{b_{1} b_{2}}-2 u^{2} K_{a_{1}}^{b_{1}} K_{a_{2}}^{b_{2}}\right) \cdots\left(\mathcal{R}_{a_{2 k-3} a_{2 k-2}}^{b_{2 k-3} b_{2 k-2}}-2 u^{2} K_{a_{2 k-3}}^{b_{2 k-3}} K_{a_{2 k-2}}^{b_{2 k-2}}\right) K_{a_{2 k 1-}}^{b_{2 k-1}} .
\end{aligned}
$$

Written explicitly, we have

$$
\begin{aligned}
L_{\text {surf }}^{(k)}= & \frac{(2 k) ! a_{(k)}}{2^{k-1}} \sum_{\ell=0}^{k-1} \frac{(k-1) !(-2)^{\ell}}{(k-\ell-1) ! \ell !(2 \ell+1)} \delta_{b_{1} \cdots b_{2 k-2 \ell-2} b_{2 k-2 \ell-1} \cdots b_{2 k-1}}^{a_{1} \cdots a_{2 k-2 \ell-2} a_{2 k-2 \ell-1} \cdots a_{2 k-1}} \times \\
& \mathcal{R}_{a_{1} a_{2}}^{b_{1} b_{2}} \cdots \mathcal{R}_{a_{2 k-2 \ell-3} b_{2 k-2 \ell-2}}^{b_{2 k-2 \ell-3} b_{2 k-2 \ell-2}} K_{a_{2 k-2 \ell-1}}^{b_{2 k-2 \ell-1}} \cdots K_{a_{2 k-1}}^{b_{2 k-1}} .
\end{aligned}
$$

Evaluating this for $k=1$ gives the standard Gibbons-Hawking boundary term for the bulk gravitational Lagrangian $a_{(1)} R$ :

$$
L_{\text {surf }}^{(1)}=2 a_{(1)} K
$$

where $K=K_{a}^{a}$, while for $k=2$ we obtain

$$
L_{\mathrm{surf}}^{(2)}=-4 a_{(2)}\left[2 \mathcal{G}_{b}^{a} K_{a}^{b}+\frac{1}{3}\left(K^{3}-3 K K_{b}^{a} K_{a}^{b}+2 K_{b}^{a} K_{c}^{b} K_{a}^{c}\right)\right]
$$

where $\mathcal{G}_{a b}=\mathcal{R}_{a b}-\frac{1}{2} \mathcal{R} h_{a b}$ is the boundary Einstein tensor. This result agrees with the surface term for Gauss-Bonnet gravity given in [38, 39]. 


\subsection{Flat-boundary counterterms for Lovelock actions}

In the previous subsection, we derived explicit general formulae for the surface terms for all Lovelock actions, with curved as well as flat boundaries. In fact, for the purposes of this paper, we are interested in the restricted results for the case where the boundary metric is flat, in which case the simple expressions given by (4.19) are sufficient. In this subsection, we shall calculate the counterterms for all Lovelock actions. Here, however, we shall restrict ourselves from the outset to the case where the boundary metric is flat, since otherwise the calculations would become to unwieldy, and we do not in any case need the counterterms involving boundary curvature for the purposes of this paper.

The counterterm action $S_{\text {ct }}$ that we seek can be determined by requiring that the leading-order power-law $r$ divergence in the total action $S_{\text {tot }}=S_{\text {bulk }}+S_{\text {surf }}+S_{\text {ct }}$ be cancelled. This will ensure that the total action is finite for $\operatorname{AdS}_{n}$ itself (corresponding to the metric (2.2) with $\tilde{f}=f=g^{2} r^{2}$ ), and this uniquely determines the general expression for the counterterms for a flat boundary metric. Note that in this $\operatorname{AdS}_{n}$ background we have $\sqrt{-g}=(g r)^{n-2}$ and $\sqrt{-h}=(g r)^{n-1}$, together with $R_{\rho \sigma}^{\mu \nu}=-2 g^{2} \delta_{\rho \sigma}^{\mu \nu}$ and $K_{b}^{a}=g \delta_{b}^{a}$.

Using (4.5), we can see that the equations of motion for the Lovelock theory with bulk action given by (4.1) imply, for the pure $\mathrm{AdS}_{n}$ background, that

$$
-\frac{1}{2} \sum_{k \geq 0} \frac{(n-1) !}{(n-2 k-1) !}\left(-g^{2}\right)^{k} a_{(k)}=0 .
$$

One may think of this equation as determining the "bare" cosmological constant $-\frac{1}{2} a_{(0)}$ in terms of the $\mathrm{AdS}_{n}$ scale-size parameter $g$, for given values of the higher Lovelock couplings $a_{(k)}$ with $k \geq 1$.

The on-shell bulk action in the $\mathrm{AdS}_{n}$ background, integrated out to a radius $\bar{r}$, is given by

$$
S_{\mathrm{bulk}}=\sum_{k \geq 0} S_{\mathrm{bulk}}^{(k)}=\int d^{n-1} x \sum_{k \geq 0} a_{(k)} \int^{\bar{r}} d r \sqrt{-g} E^{(k)},
$$

where on-shell we have, from (4.2), that

$$
E^{(k)}=\frac{n !\left(-g^{2}\right)^{k}}{(n-2 k) !}
$$

The surface terms, which can be determined from (4.19) with $K_{b}^{a}=g \delta_{b}^{a}$, give a total on-shell surface action

$$
S_{\text {surf }}=\int d^{n-1} x \sqrt{-h} \sum_{k \geq 1} L_{\text {surf }}^{(k)}, \quad L_{\text {surf }}^{(k)}=\frac{2 k g(n-1) !\left(-g^{2}\right)^{k-1} a_{(k)}}{(2 k-1)(n-2 k) !} .
$$

Evaluating $S_{\text {surf }}$ at $r=\bar{r}$, and combining it with the contribution coming from (4.25), we find that the power-law $\bar{r}$ divergence, arising at $\bar{r}^{n-1}$ order, is cancelled provided we add a counterterm action

$$
S_{\mathrm{ct}}=\int d^{n-1} x \sqrt{-h} \sum_{k \geq 1} \frac{(n-2) ! 2 k(-1)^{k} a_{(k)}}{(2 k-1)(n-2 k-1) !} g^{2 k-1} .
$$




\subsection{Boundary energy-momentum tensor}

Having obtained the complete action

$$
S_{\mathrm{tot}}=S+S_{\mathrm{ct}}, \quad S=S_{\mathrm{bulk}}+S_{\mathrm{surf}},
$$

we are now in a position to calculate the boundary energy-momentum tensor

$$
T^{a b}=\frac{2}{\sqrt{-h}} \frac{\delta S_{\mathrm{tot}}}{\delta h_{a b}}=2 \pi^{a b}+T_{\mathrm{ct}}^{a b},
$$

where

$$
\begin{aligned}
T_{\mathrm{ct}}^{a b} & =\frac{2}{\sqrt{-h}} \frac{\delta S_{\mathrm{ct}}}{\delta h_{a b}}, \\
& =\sum_{k \geq 1} \frac{(n-2) ! 2 k(-1)^{k} a_{(k)} g^{2 k-1}}{(2 k-1)(n-2 k-1) !} h^{a b} .
\end{aligned}
$$

(In obtaining the last line, we made use of the expression (4.28).)

The canonical momentum $\pi^{a b}$ is given by

$$
\pi^{a b} \equiv \frac{2}{\sqrt{-h}} \frac{\delta S}{\delta h^{a b}},
$$

evaluated on the boundary. Although we have explicitly obtained the surface action in the previous subsection 4.2 , the direct calculation of $\pi^{a b}$ from this would be somewhat involved. For a theory involving only second derivatives, it is more convenient to make use of the observation in [40], which implies that

$$
\pi^{a b}=\frac{1}{\sqrt{-h}} \frac{\delta S}{\delta \dot{h}_{a b}}=\frac{1}{2 \sqrt{-h}} \frac{\delta S}{\delta K_{a b}}=\frac{1}{2} \frac{\partial L}{\partial K_{a b}},
$$

where $L$ is defined by $S=\int d^{n} x \sqrt{-g} L$. The expression of $L$ in the ADM decomposition can best be stated in terms of the Lagendre transformation

$$
K_{a b} \frac{\partial L}{\partial K_{a b}}-L=H \equiv-L_{\mathrm{bulk}}\left(R_{c d}^{a b}\right),
$$

where the expression for the Hamiltonian $H$ in the last equality was demonstrated, for the Lovelock theories, in [41]. Here $R_{c d}^{a b}$ denotes the restriction of $R_{\rho \sigma}^{\mu \nu}$ to its components purely in the boundary directions, which are then expressed in terms of $\mathcal{R}_{c d}^{a b}$ and $K_{b}^{a}$ by using the Gauss-Codacci equations, as in (4.15). From the Legendre transformation (4.34) it follows that

$$
K_{c d} \frac{\partial \pi^{a b}}{\partial K_{c d}}=\tilde{\pi}^{a b}, \quad \text { where } \quad \tilde{\pi}^{a b}=\frac{1}{2} \frac{\partial H}{\partial K_{a b}} .
$$

The key point here is that $H\left(\mathcal{R}_{c d}^{a b}, K_{b}^{a}\right)$ is easily calculated, as in the final equality in (4.34), and hence $\tilde{\pi}^{a b}$ is easily obtained. Specifically, the contribution $H^{(k)}$ for the $k$ th Lovelock Lagrangian gives

$$
H^{(k)}=-\frac{a_{(k)}(2 k) !}{2^{k}} \delta_{b_{1} \cdots b_{2 k}}^{a_{1} \cdots a_{2 k}}\left(\mathcal{R}_{a_{1} a_{2}}^{b_{1} b_{2}}-2 K_{a_{1}}^{b_{1}} K_{a_{2}}^{b_{2}}\right) \cdots\left(\mathcal{R}_{a_{2 k-1} a_{2 k}}^{b_{2 k-1} b_{2 k}}-2 K_{a_{2 k-1}}^{b_{2 k-1}} K_{a_{2 k}}^{b_{2 k}}\right) .
$$

Then, (4.35) can be used in order to calculate $\pi^{a b}$ from $\tilde{\pi}^{a b}$. 
In our case the metric on the boundary is flat, and so $\mathcal{R}_{c d}^{a b}$ vanishes. This means that for the $k^{\prime}$ th Lovelock Lagrangian, $H^{(k)}$ given in (4.36) reduces to

$$
H^{(k)}=-(-1)^{k} a_{(k)}(2 k) ! \delta_{b_{1} \cdots b_{2 k}}^{a_{1} \cdots a_{2 k}} K_{a_{1}}^{b_{1}} \cdots K_{a_{2 k}}^{b_{2 k}} .
$$

It is homogeneous of degree $2 k$ in $K_{b}^{a}$, and hence $\tilde{\pi}_{(k)}^{a b}$ is homogeneous of degree $(2 k-1)$ in $K_{b}^{a}$. It then follows from (4.35) that

$$
\pi_{(k)}^{a b}=\frac{1}{(2 k-1)} \tilde{\pi}_{(k)}^{a b}=\frac{1}{2(2 k-1)} \frac{\partial H_{(k)}}{\partial K_{a b}} .
$$

From (4.37) we therefore have

$$
\pi_{(k) b}^{a}=-\frac{(-1)^{k} k a_{(k)}(2 k) !}{(2 k-1)} \delta_{b b_{1} \cdots b_{2 k-1}}^{a a_{1} \cdots a_{2 k-1}} K_{a_{1}}^{b_{1}} \cdots K_{a_{2 k-1}}^{b_{2 k-1}} .
$$

Evaluating first the background value of $\pi_{(k) b}^{a}$, which we shall denote by $\bar{\pi}_{(k) b}^{a}$, we find

$$
\bar{\pi}_{(k) b}^{a}=-\frac{(-1)^{k} k a_{(k)}(n-2) ! g^{2 k-1}}{(2 k-1)(n-2 k-1) !} \delta_{b}^{a} .
$$

Varying (4.39) to get the linearised perturbation in $\pi_{(k) b}^{a}$ we find

$$
\delta \pi_{(k) b}^{a}=-\frac{(-1)^{k} k a_{(k)}(n-3) !}{(n-2 k-1) !}\left(\delta K_{c}^{c} \delta_{b}^{a}-\delta K_{b}^{a}\right) .
$$

Finally, we obtain the linearised perturbation in $\delta \pi_{(k)}^{a b}$ as

$$
\delta \pi_{(k)}^{a b}=\delta \pi_{(k) c}^{a} \bar{h}^{b c}+\bar{\pi}_{(k) c}^{a} \delta h^{b c},
$$

where $\bar{h}^{a b}$ is the background value of $h^{a b}$ and $\delta h^{a b}$ is its linearised perturbation.

From $(4.30),(4.31),(4.40)$ and (4.42) we see that in calculating the linearised boundary stress tensor $T_{\text {lin }}^{a b}$, the $\bar{\pi}_{(k) c}^{a} \delta h^{b c}$ term in (4.42) is precisely cancelled by the linearised counterterm contribution $T_{\mathrm{ct}, \text { lin }}^{a b}$, and so we arrive at the final result

$$
\delta T_{(k), \operatorname{lin}}^{a b}=-\frac{(-1)^{k} 2 k a_{(k)}(n-3) ! g^{2 k-2}}{(n-2 k-1) !}\left(\delta K_{c}^{c} \delta_{b}^{a}-\delta K_{b}^{a}\right) .
$$

We are interested in comparing the expression for the $t x_{1}$ component of the linearised boundary stress tensor with the expression for the bulk Noether current $\delta J^{(k) r x_{1}}$ that we calculated in section (4.1). For our perturbed metric (2.4) we have, at the linearised order, $\delta K_{c}^{c}=0$, and $\delta K^{t}{ }_{x_{1}}=-1 /(2 g r)\left(\delta g_{t x_{1}}^{\prime}-2 \delta g_{t x_{1}} / r\right)$ and so

$$
\begin{aligned}
T_{(k), \text { lin }}^{t x_{1}} & =\frac{(-1)^{k-1} k a_{(k)}(n-3) ! g^{2 k-2}}{(n-2 k-1) !(g r)^{3}}\left(\delta g_{t x_{1}}^{\prime}-\frac{2}{r} \delta g_{t x_{1}}\right), \\
& =\frac{1}{(g r)^{n+1}} \mathcal{J}_{\operatorname{lin}}^{(k) x_{1}},
\end{aligned}
$$

where $\mathcal{J}^{(k) x_{1}}$ is the $k^{\prime}$ th term of the bulk radially-conserved Noether current obtained in eqn (4.11). Thus we have established the desired correspondence between the radially conserved bulk current and the boundary heat current. 


\section{Holographic heat current in Horndeski gravities}

In this section, we consider Einstein-Horndeski gravities, which are a class of higherderivative theories involving non-minimally coupled scalar axion fields. For simplicity, we shall include in the Lagrangian just the simplest non-trivial Horndeski term, namely [34]

$$
S=\int d^{n} x \sqrt{-g}\left(R-2 \Lambda-\frac{1}{2} \alpha \chi^{\mu} \chi_{\mu}+\frac{1}{2} \gamma G_{\mu \nu} \chi^{\mu} \chi^{\nu}\right)
$$

where $\chi$ is a scalar field, $\chi^{\mu} \equiv \partial^{\mu} \chi$, and $G_{\mu \nu}=R_{\mu \nu}-\frac{1}{2} R g_{\mu \nu}$ is the Einstein tensor. Static AdS black hole solutions with $\chi=\chi(r)$ were constructed in [42-45]. The thermodynamics of these solutions has been analysed in [46, 47]. (See also [48].)

The AdS planar black hole solution is particularly simple, and is given by

$$
\begin{aligned}
d s^{2} & =-f d t^{2}+\frac{d r^{2}}{f}+g^{2} r^{2} d x^{i} d x^{i}, & \chi & =\chi(r), \\
f & =g^{2} r^{2}-\frac{\mu}{r^{n-3}}, & \chi^{\prime} & =\sqrt{\frac{\beta}{f}} .
\end{aligned}
$$

Here a prime denotes a derivative with respect to $r$. The parameters $(\beta, g)$ are related to the coupling constants by

$$
\Lambda=-\frac{1}{2}(n-1)(n-2)\left(1+\frac{1}{2} \beta \gamma\right) g^{2}, \quad \alpha=\frac{1}{2}(n-1)(n-2) g^{2} \gamma .
$$

The solution contains only one non-trivial integration constant $\mu$, proportional to the mass of the black hole.

It follows from (2.9) that the bulk current is given by

$$
\begin{gathered}
J^{\mu \nu}=2 \nabla^{\mu} \xi^{\nu}+\gamma\left[-\frac{1}{2}(\partial \chi)^{2} \nabla^{\mu} \xi^{\nu}+\nabla^{\sigma} \chi \nabla^{[\mu} \chi \nabla_{\sigma} \xi^{\nu]}+\xi^{[\nu} \nabla^{\mu]}(\partial \chi)^{2}\right. \\
\left.-\xi^{[\nu} \nabla_{\sigma}\left(\nabla^{\mu]} \chi \nabla^{\sigma} \chi\right)-\xi^{\sigma} \nabla^{[\mu}\left(\nabla^{\nu]} \chi \nabla_{\sigma} \chi\right)\right] .
\end{gathered}
$$

Considering a linearised TT metric perturbation (2.4) and taking the Killing vector $\xi$ to be $\partial_{t}$, we find that the $r x_{1}$ component of the 2 -form $J_{(2)}$ at linear order, at large $r$, is given by

$$
J_{\text {lin }}^{r x_{1}}=\left(1+\frac{\beta \gamma}{4}\right) \frac{r \delta g_{t x_{1}}^{\prime}-2 \delta g_{t x_{1}}}{r} .
$$

Thus the radially-conserved bulk current is

$$
\delta \mathcal{J}^{x_{1}}=\sqrt{-\bar{g}} \delta J^{t x_{1}}=\sqrt{-\bar{g}}\left(1+\frac{\beta \gamma}{4}\right) \frac{r \delta g_{t x_{1}}^{\prime}-2 \delta g_{t x_{1}}}{r} .
$$

As in the case of the Lovelock gravities we discussed previously, the goal now will be to establish that the above bulk current is the holographic dual to the boundary heat current, i.e. that it matches to the boundary stress tensor. 
The generalized Gibbons-Hawking term is

$$
S_{\text {surf }}=4 \int d x^{n-1} \sqrt{-h} \frac{\partial L}{\partial R_{\rho \sigma}^{\mu \nu}} K_{\rho}^{\mu} n^{\mu} n_{\sigma}=2 \int d x^{n-1} \sqrt{-h} K .
$$

In other words, the Horndeski term does not modify the surface term. As in the previous section on Lovelock theories, we can use the Gauss-Codacci relation to express the bulk curvature in terms of the intrinsic boundary curvature $\mathcal{R}_{c d}^{a b}$ and the extrinsic curvature $K_{b}^{a}$ :

$$
\begin{aligned}
G_{\mu \nu} n^{\mu} n^{\nu} & =-\frac{1}{2} h^{\mu \nu} h^{\rho \sigma} R_{\mu \rho \nu \sigma}=-\frac{1}{2}\left(\mathcal{R}-K^{2}+K_{a b}^{2}\right), \\
R & =\mathcal{R}+K^{2}-K_{a b}^{2}+2 \nabla_{\alpha}\left(n^{\beta} \nabla_{\beta} n^{\alpha}-n^{\alpha} \nabla_{\beta} n^{\beta}\right) .
\end{aligned}
$$

For the AdS planar black hole in Horndeski gravity we considered above, the scalar axion $\chi$ depends only on the coordinate $r$, and the Horndeski term can be thus written as

$$
G^{\mu \nu} \chi_{\mu} \chi_{\nu}=G_{\mu \nu} \chi_{\rho} \chi_{\sigma} n^{\mu} n^{\nu} n^{\rho} n^{\sigma}=-\frac{1}{2}\left(\mathcal{R}-K^{2}+K_{a b}^{2}\right) \chi^{r} \chi_{r}
$$

Indeed, this result is consistent with the earlier observation that there is no GibbonsHawking type surface contribution associated with the Horndeski term.

The total bulk action together with Gibbons-Hawking surface term, expressed in terms of the induced metric and exterior curvature $K_{\mu \nu}$, is given by

$$
S_{\mathrm{bulk}}+S_{\mathrm{surf}}=\int d^{n} x \sqrt{-g}\left[\left(1+\frac{1}{4} \gamma \chi^{r} \chi_{r}\right)\left(K^{2}-K_{a b}^{2}\right)-2 \Lambda-\frac{\alpha}{2} \chi^{\mu} \chi_{\mu}\right] .
$$

Note that the intrinsic curvature contribution vanishes, i.e. $\mathcal{R}=0$, since the boundary of the AdS planar black hole we consider is flat. The corresponding Hamiltonian is then given by

$$
\mathcal{H}=K_{a b} \frac{\partial L}{K_{a b}}-L=\left(1+\frac{1}{4} \gamma \chi^{r} \chi_{r}\right)\left(K^{2}-K_{a b}^{2}\right),
$$

from which we obtain the canonical momentum

$$
\pi^{a b}=\frac{1}{2} \frac{\partial \mathcal{H}}{\partial K_{a b}}=\left(1+\frac{1}{4} \gamma \chi^{r} \chi_{r}\right)\left(K h^{a b}-K^{a b}\right) .
$$

The counterterm for this theory can be obtained by using the same strategy as in our discussion of the Lovelock gravities. It was in fact obtained in [46], and is given by

$$
S_{\mathrm{ct}}=-\int d x^{n-1} \sqrt{h} 2(n-2)\left(1+\frac{1}{4} \beta \gamma\right) g .
$$

The contribution to the boundary stress tensor from the counterterm is

$$
T_{\mathrm{ct}}^{a b}=\frac{2}{\sqrt{-h}} \frac{\delta S_{c t}}{\delta h_{a b}}=-2(n-2)\left(1+\frac{1}{4} \beta \gamma\right) g h^{a b} .
$$

The full boundary stress tensor is therefore given by

$$
T^{a b}=2 \pi^{a b}+T_{\mathrm{ct}}^{a b}=2\left(1+\frac{1}{4} \gamma \chi^{r} \chi_{r}\right)\left(g h^{a b}-K^{a b}\right) .
$$


The $t x_{1}$ component of the stress tensor, to linear order in the perturbation, is then given by

$$
T_{\text {lin }}^{t x_{1}}=\left(1+\frac{1}{4} \beta \gamma\right) \frac{r \delta g_{t x_{1}}^{\prime}-2 g_{t x_{1}}}{g^{3} r^{4}} .
$$

The corresponding heat current is

$$
\mathcal{Q}^{x_{1}}=(g r)^{n+1} T_{\text {lin }}^{t x_{1}}=\left(1+\frac{1}{4} \beta \gamma\right) g^{n-2} r^{n-3}\left(r \delta g_{t x_{1}}^{\prime}-2 \delta g_{t x_{1}}\right) .
$$

It can easily be seen that it matches precisely on the boundary with the radially-conserved Noether current $\delta \mathcal{J}^{x_{1}}$ given in (5.6). Note that although we established the matching of the bulk Noether current and boundary heat current using the explicit AdS planar black hole solution, the matching works even for more general black holes with additional matter fields, as long as the asymptotic metric functions take the form $\tilde{f} \sim f \sim g^{2} r^{2}$ at large $r$.

Einstein-Horndeski gravities with multiple Horndeski axions can also admit a different class of black hole solutions, in which the axions play the direct role of providing the momentum dissipation. The Lagrangian is given by

$$
\frac{\mathcal{L}}{\sqrt{-g}}=R-2 \Lambda-\frac{1}{4} F^{2}-\frac{1}{2}\left(\alpha g^{\mu \nu}-\gamma G^{\mu \nu}\right) \sum_{i=1}^{N} \partial_{\mu} \chi_{i} \partial_{\nu} \chi_{i}
$$

The ansatz for the AdS planar black holes is

$$
\begin{aligned}
d s_{n}^{2} & =-h(r) d t^{2}+\frac{d r^{2}}{f(r)}+r^{2}\left(d x_{1}^{2}+\cdots+d x_{n-2}^{2}\right), \\
A & =a(r) d t, \quad \chi_{i}= \begin{cases}\lambda x_{i}, & i=1,2, \ldots, n-2, \\
0, & i \geq n-1\end{cases}
\end{aligned}
$$

The four-dimensional solution was constructed in [27], and the higher-dimensional generalizations were given in [49]. The holographic DC conductivities were analysed in [27]. The four-dimensional radially-conserved bulk current associated with the holographic heat current was given in [28], although its match with the boundary stress tensor was not demonstrated.

It follows from (2.9) and (2.17) that the radially-conserved Noether current at large $r$ is given by

$$
\mathcal{J}_{\operatorname{lin}}^{x_{1}}=\sqrt{-\bar{g}}\left(r \delta g_{t x_{1}}^{\prime}-2 g_{t x_{1}}\right)\left(\frac{1}{r}-\frac{n-4}{4 r^{3}} \gamma \lambda^{2}\right) .
$$

It is evident that the Horndeski term contributes a sub-leading order relative to the contribution from the Einstein-Hilbert term on the boundary, and hence it can be neglected. The same is true for the boundary stress tensor, as can be seen on the grounds of dimensional analysis; namely, the quantity $\gamma \lambda^{2}$ has dimension of length squared. It then follows that the matching (2.19) holds straightforwardly. 


\section{Conclusions}

A radially conserved current in the bulk that matches the boundary heat current is a key ingredient in the holographic study of thermal conductivity and related transport coefficients. It allows one to read off the relevant transport properties directly and analytically from the black hole horizon data of the solution in the bulk theory. In this paper, we employed a Noether procedure, closely related to that used by Wald [31, 32], and derived a formula (2.17) for a radially conserved bulk current for a general class of gravity theories, in the case of AdS planar black hole backgrounds (2.2) with a TT perturbation of the form (2.4). We showed that the formula (2.17) reproduced known results in literature, including the EMD theory [15], Einstein-Gauss-Bonnet gravity [19] and also four-dimensional Eintein-Horndeski gravity [28].

In order to demonstrate that the Noether current (2.17) is indeed the holographic bulk dual to the heat current, it is necessary to show that it matches the boundary heat current derived from the boundary stress tensor. We focused our discussion on the general class of all Lovelock higher-derivative gravities. We derived the generalized Gibbons-Hawking surface term for the general Lovelock gravities, and we constructed the boundary counterterms for the flat boundaries that arise for AdS planar black holes. This enabled us to construct the full boundary stress tensor and to derive the heat current associated with TT perturbations. We showed that the bulk Noether current and the boundary heat current match precisely. We also performed an analogous analysis for a class of Horndeski gravities in general dimensions, and showed again that the Noether current (2.17) is the relevant radially-conserved current that describes the holographic heat current.

Our discussion was concerned only with ghost-free theories such as Lovelock and Horndeski gravities. Even though the total number of derivatives in each term in the equations of motion can exceed two this occurs only because of the nonlinearities; the linearized equations of motion around any background involve only two derivatives at most. This makes it easier to construct the Hamiltonian and to derive the boundary stress tensor. Indeed, as we have shown in these cases the Noether current (2.17) matches precisely the heat current derived from the boundary stress tensor.

The Noether current (2.17) on the other hand is radially conserved for more general classes of gravity theories that may have ghost excitations as well. We expect that it is still the relevant holographic bulk dual to the heat current even in these more general situations, but this remains to be investigated in detail.

\section{Acknowledgments}

We thank Aristos Donos, Luis Fernando, Jerome Gauntlett and Tom Griffin for useful comments on our paper. C.N.P. is grateful to the Center for Advanced Quantum Studies and the physics department at Beijing Normal University for hospitality during the course of this work. H-S.L. is supported in part by NSFC grants No. 11305140, No. 11375153, No. 11475148 and No. 11675144. H.L. is supported in part by NSFC grants No. 11475024, No. 11175269 and No. 11235003. C.N.P. is supported in part by DOE grant DE-FG0213ER42020. 
Open Access. This article is distributed under the terms of the Creative Commons Attribution License (CC-BY 4.0), which permits any use, distribution and reproduction in any medium, provided the original author(s) and source are credited.

\section{References}

[1] S.A. Hartnoll, Lectures on holographic methods for condensed matter physics, Class. Quant. Grav. 26 (2009) 224002 [arXiv:0903.3246] [INSPIRE].

[2] S. Sachdev, What can gauge-gravity duality teach us about condensed matter physics?, Ann. Rev. Condensed Matter Phys. 3 (2012) 9 [arXiv:1108.1197] [INSPIRE].

[3] J. McGreevy, TASI 2015 lectures on quantum matter (with a view toward holographic duality), arXiv: 1606.08953 [INSPIRE].

[4] J. Zaanen, Y.W. Sun, Y. Liu and K. Schalm, Holographic duality in condensed matter physics, Cambridge University Press, Cambridge U.K. (2015).

[5] G.T. Horowitz, J.E. Santos and D. Tong, Optical conductivity with holographic lattices, JHEP 07 (2012) 168 [arXiv:1204.0519] [INSPIRE].

[6] G.T. Horowitz, J.E. Santos and D. Tong, Further evidence for lattice-induced scaling, JHEP 11 (2012) 102 [arXiv: 1209.1098] [INSPIRE].

[7] G.T. Horowitz and J.E. Santos, General relativity and the cuprates, JHEP 06 (2013) 087 [arXiv: 1302.6586] [INSPIRE].

[8] R.A. Davison, Momentum relaxation in holographic massive gravity, Phys. Rev. D 88 (2013) 086003 [arXiv: 1306.5792] [InSPIRE].

[9] P. Chesler, A. Lucas and S. Sachdev, Conformal field theories in a periodic potential: results from holography and field theory, Phys. Rev. D 89 (2014) 026005 [arXiv:1308.0329] [INSPIRE].

[10] M. Blake and D. Tong, Universal resistivity from holographic massive gravity, Phys. Rev. D 88 (2013) 106004 [arXiv: 1308.4970] [InSPIRE].

[11] Y. Ling, C. Niu, J.-P. Wu and Z.-Y. Xian, Holographic lattice in Einstein-Maxwell-Dilaton gravity, JHEP 11 (2013) 006 [arXiv:1309.4580] [INSPIRE].

[12] A. Donos and J.P. Gauntlett, Holographic Q-lattices, JHEP 04 (2014) 040 [arXiv:1311.3292] [INSPIRE].

[13] T. Andrade and B. Withers, A simple holographic model of momentum relaxation, JHEP 05 (2014) 101 [arXiv: 1311.5157] [INSPIRE].

[14] A. Donos and J.P. Gauntlett, Novel metals and insulators from holography, JHEP 06 (2014) 007 [arXiv: 1401.5077] [INSPIRE].

[15] A. Donos and J.P. Gauntlett, Thermoelectric DC conductivities from black hole horizons, JHEP 11 (2014) 081 [arXiv:1406.4742] [INSPIRE].

[16] A. Donos and J.P. Gauntlett, The thermoelectric properties of inhomogeneous holographic lattices, JHEP 01 (2015) 035 [arXiv:1409.6875] [INSPIRE].

[17] Y. Ling, P. Liu, C. Niu, J.-P. Wu and Z.-Y. Xian, Holographic superconductor on Q-lattice, JHEP 02 (2015) 059 [arXiv: 1410.6761] [InSPIRE]. 
[18] M. Baggioli and O. Pujolàs, Electron-phonon interactions, metal-insulator transitions and holographic massive gravity, Phys. Rev. Lett. 114 (2015) 251602 [arXiv:1411.1003] [INSPIRE].

[19] L. Cheng, X.-H. Ge and Z.-Y. Sun, Thermoelectric DC conductivities with momentum dissipation from higher derivative gravity, JHEP 04 (2015) 135 [arXiv:1411.5452] [INSPIRE].

[20] A. Donos and J.P. Gauntlett, Navier-Stokes equations on black hole horizons and DC thermoelectric conductivity, Phys. Rev. D 92 (2015) 121901 [arXiv:1506.01360] [INSPIRE].

[21] E. Banks, A. Donos and J.P. Gauntlett, Thermoelectric DC conductivities and Stokes flows on black hole horizons, JHEP 10 (2015) 103 [arXiv:1507.00234] [INSPIRE].

[22] A. Donos, J.P. Gauntlett, T. Griffin and L. Melgar, DC conductivity of magnetised holographic matter, JHEP 01 (2016) 113 [arXiv:1511.00713] [INSPIRE].

[23] X.-H. Ge, Y. Tian, S.-Y. Wu and S.-F. Wu, Hyperscaling violating black hole solutions and Magneto-thermoelectric DC conductivities in holography, Phys. Rev. D 96 (2017) 046015 [arXiv: 1606.05959] [INSPIRE].

[24] X.-H. Ge, Y. Tian, S.-Y. Wu, S.-F. Wu and S.-F. Wu, Linear and quadratic in temperature resistivity from holography, JHEP 11 (2016) 128 [arXiv:1606.07905] [INSPIRE].

[25] S. Cremonini, H.-S. Liu, H. Lü and C.N. Pope, DC conductivities from non-relativistic scaling geometries with momentum dissipation, JHEP 04 (2017) 009 [arXiv:1608.04394] [INSPIRE].

[26] A. Donos, J.P. Gauntlett, T. Griffin and L. Melgar, DC conductivity and higher derivative gravity, Class. Quant. Grav. 34 (2017) 135015 [arXiv:1701.01389] [INSPIRE].

[27] W.-J. Jiang, H.-S. Liu, H. Lü and C.N. Pope, DC conductivities with momentum dissipation in Horndeski theories, JHEP 07 (2017) 084 [arXiv: 1703.00922] [INSPIRE].

[28] M. Baggioli and W.-J. Li, Diffusivities bounds and chaos in holographic Horndeski theories, JHEP 07 (2017) 055 [arXiv: 1705.01766] [INSPIRE].

[29] N. Iqbal and H. Liu, Universality of the hydrodynamic limit in AdS/CFT and the membrane paradigm, Phys. Rev. D 79 (2009) 025023 [arXiv:0809.3808] [INSPIRE].

[30] Y.-Z. Li, H.-S. Liu and H. Lü, Quasi-Topological Ricci Polynomial Gravities, arXiv:1708.07198 [INSPIRE].

[31] R.M. Wald, Black hole entropy is the Noether charge, Phys. Rev. D 48 (1993) R3427 [gr-qc/9307038] [INSPIRE].

[32] V. Iyer and R.M. Wald, Some properties of Noether charge and a proposal for dynamical black hole entropy, Phys. Rev. D 50 (1994) 846 [gr-qc/9403028] [INSPIRE].

[33] D. Lovelock, The Einstein tensor and its generalizations, J. Math. Phys. 12 (1971) 498 [INSPIRE].

[34] G.W. Horndeski, Second-order scalar-tensor field equations in a four-dimensional space, Int. J. Theor. Phys. 10 (1974) 363 [INSPIRE].

[35] J.W. York, Jr., Role of conformal three geometry in the dynamics of gravitation, Phys. Rev. Lett. 28 (1972) 1082 [INSPIRE].

[36] G.W. Gibbons and S.W. Hawking, Action integrals and partition functions in quantum gravity, Phys. Rev. D 15 (1977) 2752 [InSPIRE]. 
[37] N. Deruelle, M. Sasaki, Y. Sendouda and D. Yamauchi, Hamiltonian formulation of f(Riemann) theories of gravity, Prog. Theor. Phys. 123 (2010) 169 [arXiv:0908.0679] [INSPIRE].

[38] R.C. Myers, Higher derivative gravity, surface terms and string theory, Phys. Rev. D 36 (1987) 392 [InSPIRE].

[39] J.T. Liu and W.A. Sabra, Hamilton-Jacobi counterterms for Einstein-Gauss-Bonnet gravity, Class. Quant. Grav. 27 (2010) 175014 [arXiv:0807.1256] [INSPIRE].

[40] J. de Boer, E.P. Verlinde and H.L. Verlinde, On the holographic renormalization group, JHEP 08 (2000) 003 [hep-th/9912012] [INSPIRE].

[41] C. Teitelboim and J. Zanelli, Dimensionally continued topological gravitation theory in Hamiltonian form, Class. Quant. Grav. 4 (1987) L125 [INSPIRE].

[42] A. Cisterna and C. Erices, Asymptotically locally AdS and flat black holes in the presence of an electric field in the Horndeski scenario, Phys. Rev. D 89 (2014) 084038 [arXiv: 1401.4479] [INSPIRE].

[43] A. Anabalon, A. Cisterna and J. Oliva, Asymptotically locally AdS and flat black holes in Horndeski theory, Phys. Rev. D 89 (2014) 084050 [arXiv:1312.3597] [INSPIRE].

[44] M. Rinaldi, Black holes with non-minimal derivative coupling, Phys. Rev. D 86 (2012) 084048 [arXiv:1208.0103] [INSPIRE].

[45] E. Babichev and C. Charmousis, Dressing a black hole with a time-dependent Galileon, JHEP 08 (2014) 106 [arXiv: 1312.3204] [INSPIRE].

[46] X.-H. Feng, H.-S. Liu, H. Lü and C.N. Pope, Black hole entropy and viscosity bound in Horndeski gravity, JHEP 11 (2015) 176 [arXiv:1509.07142] [INSPIRE].

[47] X.-H. Feng, H.-S. Liu, H. Lü and C.N. Pope, Thermodynamics of charged black holes in Einstein-Horndeski-Maxwell theory, Phys. Rev. D 93 (2016) 044030 [arXiv:1512.02659] [INSPIRE].

[48] E. Caceres, R. Mohan and P.H. Nguyen, On holographic entanglement entropy of Horndeski black holes, arXiv: 1707.06322 [INSPIRE].

[49] X.-H. Feng, H.-S. Liu, W.-T. Lu and H. Lü, Horndeski gravity and the violation of reverse isoperimetric inequality, arXiv:1705.08970 [INSPIRE]. 